\title{
Three-dimensional model of x-ray induced microchannel plate output
}

\author{
E. C. Harding and R. P. Drake \\ University of Michigan, 2455 Hayward Street, Ann Arbor, Michigan 48109
}

(Received 8 May 2006; presented on 8 May 2006; accepted 29 May 2006; published online 2 October 2006)

\begin{abstract}
Microchannel plates are an important component in a type of imaging diagnostic known as an x-ray framing camera, used in x-ray radiography of high-energy-density physics experiments. A microchannel plate is responsible for detecting $\mathrm{x}$ rays and then converting them into amplified bursts of electrons, which are then imaged onto a phosphor-coated fiber optic screen. We present the preliminary development of a three-dimensional model of a single microchannel plate channel in attempt to simulate the pulse height distribution of the microchannel plate electron output. Using a novel technique, initial simulations are compared with experimental data from an ungated x-ray framing camera. @ 2006 American Institute of Physics. [DOI: 10.1063/1.2220072]
\end{abstract}

\section{INTRODUCTION}

Gated x-ray framing cameras are the primary detectors used in x-ray radiography of evolving hydrodynamic flows in high-energy-density experiments. The detailed operation of gated framing cameras has been previously investigated elsewhere. ${ }^{1-4}$ Here we are concerned with the detailed operation of the x-ray detection and electron amplification processes of the individual channels that make up the microchannel plate (MCP). The astrophysical uses of MCPs involve pulse counting, and extensive work has been done on such applications. ${ }^{5-7}$ In such applications, variations in the gain of the channel from pulse to pulse are not important. In contrast, the high-energy-density applications typically involve using MCPs as amplifiers for analog imaging, producing images having several to very many detected photons per resolution element. An ideal amplifier for such an application would produce an identical output pulse for each input photon. Instead, a MCP actually produces a broad pulse height distribution at its output, which contributes substantially to the noise in the image. This motivates work to understand and then to optimize this pulse height distribution. Here we report the initial development of a model intended to contribute to this effort.

Beyond the general value of understanding and improving MCP performance, a review of the framing camera literature reveals some specific useful topics of study. For instance, MCP channels that have square cross sections, instead of the traditional cylindrical ones, may yield less channel-to-channel variation of the MCP electron output by establishing a uniform angle of incidence. Furthermore, the effect of the fringing electric fields, which make up the "endspoiled" region of the channel input, has not been well quantified. Although a rough estimation of the "end-spoiling" effect has been used previously to improve the fit of experimental data and theory, no complete physical reasoning has been presented. ${ }^{8,9}$ In addition, when applying a high quantum efficiency photocathode, such as CsI, to the MCP input area one would also like to determine the required thickness and coating depth down a channel to produce the desired MCP output. In some cases, $\mathrm{x}$-ray detection may be of primary importance while in other cases, the control of the shape of the pulse height distribution may be more important. Whatever the case may be a reliable model is needed to predict the performance of the MCP without costly laboratory tests. To our knowledge, no one has successfully constructed a three-dimensional (3D) MCP model that simulates the absolute output pulse height distribution (PHD). We report our work in that direction here.

\section{THEORY}

Our model assumes that every channel on a single MCP has identical material composition and electrode/ photocathode coating specifications (e.g., coating thickness and coating depth down a channel). Therefore, the activation of many channels, spatially distributed across the input surface of the MCP, can be modeled as a single channel struck by many photons. Our present model does not yet account for multiple channel crossings of a single $\mathrm{x}$ ray, and thus only valid for $\mathrm{x}$ ray energies that have a mean free path much less than the wall thickness separating adjacent channels, which is $\sim 5 \mathrm{keV}$ for a $2 \mu \mathrm{m}$ wall. ${ }^{8}$ Figure 1(a) shows the geometry of the single channel used in our working model. Notice that the opening of the channel, at the input surface plane, is slightly elliptical due to the manufacturing process during which the MCP is cut at an angle from a cylindrical boule of MCP material in order to form channels with a specific bias angle.

The x-ray flux used in the model is assumed to be nondivergent and normally incident to the MCP surface, as seen in Fig. 1(a). Typically the x rays used in high-energy-density experiments are generated by line emission, so the model $\mathrm{x}$-ray flux is assumed to be monochromatic.

The model begins by randomly placing a specified number of incident $\mathrm{x}$-ray photons on the input surface plane of the channel. The position of the photons on the channel wall, given by coordinates $\left(x_{s}, y_{s}, z_{s}\right)$ in Fig. 1(a), and the corresponding grazing incident angles $(\alpha)$ are then determined. $\alpha$ is given by 
(a)

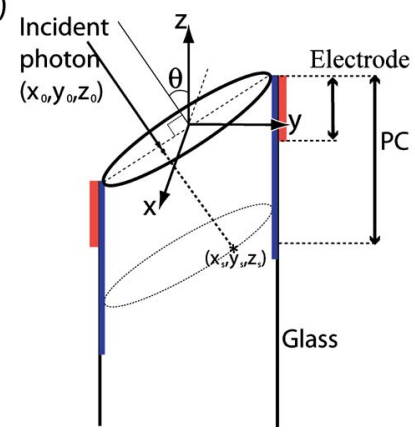

(b)

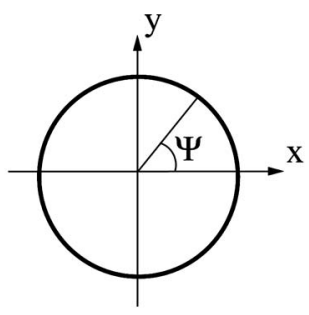

FIG. 1. (Color online) Geometry of the model MCP channel. PC =photocathode.

$\sin \alpha=\cos \psi \sin \theta$,

where $\theta$ is the channel bias angle and $\psi$ is the channel polar angle, as defined in Fig. 1(b). ${ }^{6}$ Thus, $0 \leqslant \alpha \leqslant \theta$. It is important to note that the variation of $\alpha$ is purely a $3 \mathrm{D}$ effect and is therefore not contained in two-dimensional (2D) models. As will be shown shortly, $\alpha$ is an important parameter in determining the secondary electron yield of the incident $\mathrm{x}$ rays.

Once the position of an X-ray strike is calculated, the model then determines what type and combination of materials the $\mathrm{x}$ ray has struck. There are four possibilities: (1) MCP glass, (2) conducting electrode, which is typically nichrome, or if the electrode is also serving as the photocathode a popular choice is gold, (3) insulating photocathode material, such as $\mathrm{CsI}$ or $\mathrm{KBr}$, with no electrode undercoat, and (4) insulating photocathode with an electrode undercoat. Due to the end-spoiling effect, in which secondary electrons born into the region of the MCP coated with a conducting electrode may experience gain suppression, it is important to distinguish between cases (3) and (4). In the current model the secondary electron contribution from the interface of two materials, such as MCP glass and an insulating photocathode coating, is assumed to be negligible due to the relatively short escape lengths of secondary electrons compared to the coating thickness.

Next, the mean secondary electron yield of the x-ray/ material interaction is calculated using a semiempirical model developed by Fraser. ${ }^{10}$ The primary electron yield is assumed to be negligible compared to the secondary electron yield. ${ }^{11}$ The mean secondary electron yield (SEY) is given by

$$
\delta=[1-R(\alpha)](1-f) P_{s}(0) E_{x} \varepsilon^{-1}(1+\beta)^{-1} Y(T),
$$

where $\beta=\left[\mu L_{s} \csc \left(\alpha^{\prime}\right)\right]^{-1}$ and $Y(T)$ is the relative yield versus thickness function

$$
Y(T)=1-\exp \left[-T\left(\mu \csc \alpha^{\prime}+L_{s}^{-1}\right)\right] .
$$

$R(\alpha)$ is the grazing incident dependent Fresnel reflection coefficient, $f$ is the fraction of $\mathrm{x}$-ray energy lost to primary electrons and material fluoresce, $P_{s}(0)$ is the average probability that a secondary electron will escape into vacuum once it has reached the surface of the material, $E_{x}$ is the x-ray photon energy, $\varepsilon$ is the average energy required to create a single secondary electron in the bulk of the material, $\mu$ is the $\mathrm{x}$-ray linear absorption coefficient, $L_{s}$ is the average second-

TABLE I. Required secondary electron parameters for the SEY model.

\begin{tabular}{lllc}
\hline \hline & $P_{s}(0)$ & $\begin{array}{c}L_{s} \\
(\AA)\end{array}$ & $\begin{array}{c}\varepsilon \\
(\mathrm{eV})\end{array}$ \\
\hline MCP glass $^{\mathrm{a}}$ & 0.15 & 33 & 10.0 \\
Nichrome $^{\mathrm{a}}$ & 0.024 & 20 & 6.65 \\
$\mathrm{Au}^{\mathrm{b}}$ & 0.03 & 20 & 8.0 \\
\hline \hline
\end{tabular}

${ }^{\mathrm{a}}$ Reference 10 .

${ }^{\mathrm{b}}$ Reference 12 .

ary electron escape depth, $\alpha^{\prime}$ is the angle of refraction of the incident $\mathrm{x}$ ray, and $T$ is the thickness of the material struck by the $\mathrm{x}$ ray. The parameters $P_{s}(0), L_{s}$, and $\varepsilon$ are taken to be semiempirical constants for each material type (see Table I for examples).

The values $R(\alpha), \alpha^{\prime}$, and $\mu$ are calculated using the Henke X-ray tables. ${ }^{13,14}$ Figure 2 shows grazing incident angle dependence of the SEY for $E_{x}=1.49 \mathrm{keV}(\mathrm{Al} K \alpha)$ for materials listed in Table I.

The SEY value to be used for each specific photon in the MCP model is found by randomly sampling a Poisson distribution with mean $\delta$. The position dependent gain $G(h)$ experienced by these electrons is approximated by the discrete dynode model $^{15}$

$$
G(h)=\left(\frac{V}{V_{0}}\right)^{L / 4 D[1-(h / L)]},
$$

where $V$ is the voltage applied to the MCP, $V_{0}$ is the voltage required for unity gain, $L$ is the channel length, $D$ is the channel diameter, and $h$ is the distance down the channel that the secondary electrons are born. Thus, the product of the randomly sampled SEY and $G(h)$ determines the electron output of the channel model.

\section{EXPERIMENT}

We used a dc x-ray source (Manson type) to irradiate an $\mathrm{X}$-ray framing camera operated with static voltages applied to both the MCP and the phosphor screen. The X-ray source creates two identical $\mathrm{x}$-ray beams with one directed at the framing camera and the other at a calibrated $\mathrm{x}$-ray photodiode (IRD AXUV-100). When the framing camera and $\mathrm{x}$-ray

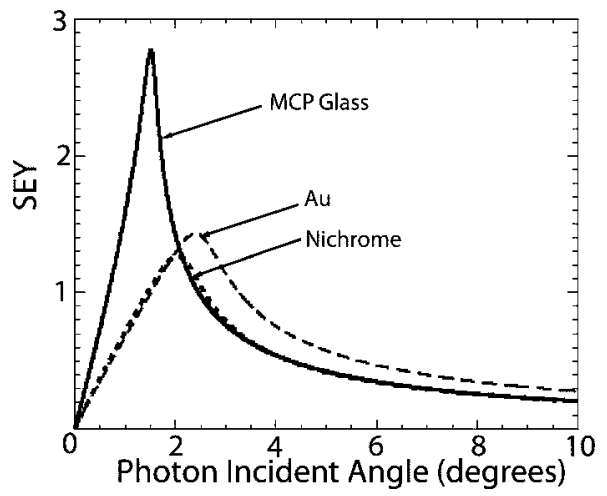

FIG. 2. Grazing incident angle dependence of SEY for a planar sample of MCP glass, nichrome, and Au using the Fraser model (Ref. 6). $f=0$ for all plotted values. The MCP glass composition used for the SEY calculations is the type produced by Galileo given in Ref. 6 . 


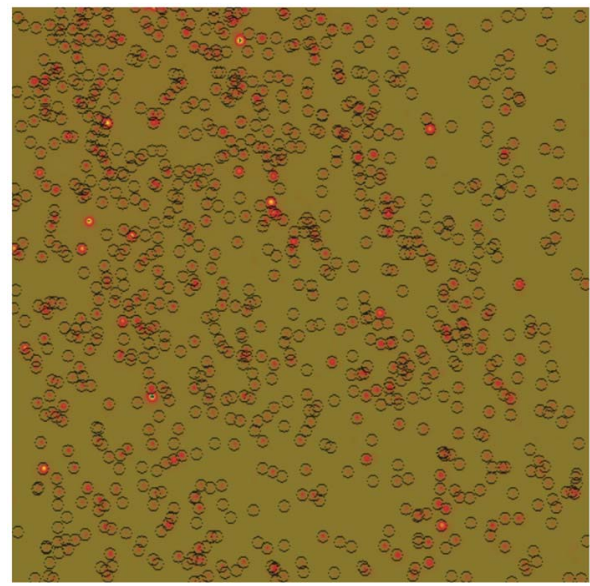

FIG. 3. (Color online) A false color image of single MCP output events due to the detection of single x-ray photons. For this image: applied MCP voltage is $1100 \mathrm{~V}$, applied phosphor voltage is $4 \mathrm{kV}, \mathrm{CCD}$ exposure time is $10 \mathrm{~ms}$, and x-ray flux is $2.4 \pm 0.1$ photons/s/channel, presumably from the $\mathrm{Al} K \alpha(1.49 \mathrm{keV})$. A star finding routine called FIND, taken from the online IDL Astronomy User's Library, was used to find and record the brightness of each event. Circles indicate a found event. The MCP used here is a Burle Long-Life ${ }^{\mathrm{TM}}$ MCP with $L / D=60, D=10 \mu \mathrm{m}, \theta=8^{\circ}$, and a nichrome (80/20) electrode coating that penetrates $\sim 5 \mu \mathrm{m}$ down the channel. There is no insulating photocathode coating. Since the average channel recharge time is $\sim 10 \mathrm{~ms}$, the MCP is likely to be operating in an unsaturated regime (Ref. 2).

source are operated in a regime where the photon fluence per channel is $\ll 1$ and the MCP gain is high $(V \sim 1 \mathrm{kV})$, the phosphor screen becomes spotted with single MCP output events, as seen in Fig. 3.

A PHD can be constructed from Fig. 3 and compared with the model PHD, by striking our model channel with the same number of photons that are incident on the open area fraction of the MCP (i.e., assume the photons that strike the interchannel space go undetected), in the field of view of the charge coupled device (CCD). Figure 4 shows the resulting

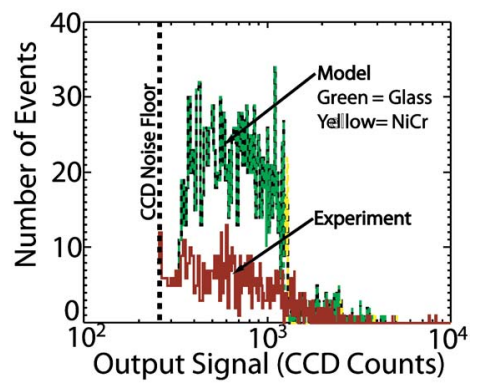

FIG. 4. (Color online) A comparison of the PHD from our MCP channel model and experiment. From the photodiode measurement we estimate that $8610 \pm 400$ photons strike the MCP open area in the CCD field of view. Thus the model simulates 8600 incident photons each with $1.49 \mathrm{keV}$. The experimental PHD was taken from the data in Fig. 3. The model output is scaled by a factor of 0.01 to account the conversion of electrons to visible light at the phosphor screen, scattering losses in the fiber optic and CCD coupling lens, and CCD digitization. Events that occur in the first half of the electrode region are neglected due to end spoiling. Model SEY values are taken from Fig. 3 and $V_{0}=500 \mathrm{~V}$. comparison of the simulated and experimental PHD.

The model in its present, preliminary form appears to capture the range of intensity of output events but overestimates the quantum efficiency of the MCP glass. We are now proceeding to improve the measurements by checking the source spectrum, confirming the diode calibration, obtaining new MCPs (the one used for Fig. 4 was old), and increasing the sensitivity of the system. We also need to assess the actual composition of the MCP glass. In addition, we are further scrutinizing and improving the model. Future improvements include an accurate treatment of end spoiling based on electron optics simulations in actual fields and random sampling of the gain distribution, reflecting the statistical nature of the gain process. ${ }^{16}$

\section{SUMMARY}

We have reported the initial development of a 3D model of the pulse height distribution produced by a microchannel plate. The model accounts for the actual variation in angle of incidence and the fact that different photons strike different materials. It also treats the statistical variation in secondary electron yield with angle of incidence and for the variation of average channel gain with electron depth. In comparison with very preliminary experiments, the model accurately captures the range of pulse heights but appears to significantly overpredict the number of pulses produced by the MCP glass. Future work will involve improvements to the experiments and the model.

\section{ACKNOWLEDGMENTS}

The authors would like to acknowledge useful technical discussions with Jim Weaver and Yefim Aglitskiy. This work is supported by the Naval Research Laboratory in Washington, DC under Contract No. N00173-05-1-G903.

${ }^{1}$ C. J. Pawley and A. V. Deniz, Rev. Sci. Instrum. 71, 1286 (2000).

${ }^{2}$ J. D. Kilkenny, Laser Part. Beams 9, 49 (1991).

${ }^{3}$ K. S. Budil, T. S. Perry, and P. M. Bell, Rev. Sci. Instrum. 67, 485 (1996).

${ }^{4}$ J. D. Wiedwald, P. M. Bell, R. Costa, J. D. Kilkenny, R. Bonner, and D. S. Montgomery, Proc. SPIE 1346, 449 (1996).

${ }^{5}$ G. W. Fraser, M. A. Barstow, M. J. Whiteley, and A. Wells, Nature (London) 300, 509 (1982).

${ }^{6}$ G. W. Fraser, Nucl. Instrum. Methods Phys. Res. 195, 523 (1982).

${ }^{7}$ G. W. Fraser, Nucl. Instrum. Methods Phys. Res. A 471, 170 (2001).

${ }^{8}$ O. L. Landen, A. Lobban, T. Tutt, P. M. Bell, R. Costa, D. R. Hargrove, and F. Ze, Rev. Sci. Instrum. 72, 709 (2001).

${ }^{9}$ D. G. Simons, G. W. Fraser, P. A. J. De Korte, J. F. Pearson, and L. De Jong, Nucl. Instrum. Methods Phys. Res. A 261, 579 (1987).

${ }^{10}$ G. W. Fraser, Nucl. Instrum. Methods Phys. Res. 206, 251 (1983).

${ }^{11}$ B. L. Henke, J. P. Knauer, and K. Premaratne, J. Appl. Phys. 52, 1509 (1981).

${ }^{12}$ G. W. Fraser, M. T. Pain, J. E. Lees, C. R. Binns, J. F. Pearson, and P. R. Houghton, Nucl. Instrum. Methods Phys. Res. A 321, 385 (1992).

${ }^{13}$ B. L. Henke, Phys. Rev. A 6, 94 (1972).

${ }^{14}$ B. L. Henke, E. M. Gullikson, and J. C. Davis, At. Data Nucl. Data Tables 54, 181 (1993).

${ }^{15}$ E. H. Eberhardt, Appl. Opt. 18, 1418 (1979).

${ }^{16}$ R. L. Bell, Exp. Tech. Phys. (Berlin) ED-22, 821 (1975). 